\title{
Short Range Order in Polymers within Neutrons' Eyeshot
}

\author{
W. ZAJA̧C \\ The Henryk Niewodniczański Institute of Nuclear Physics, PAS \\ Radzikowskiego 152, 31-342 Kraków, Poland
}

\begin{abstract}
The paper presents a short review of neutron scattering results obtained during investigation of short range order in bulk polymers. Neutron scattering proves exceptionally powerful in this type of study through (i) large penetration depth of bulk samples, (ii) isotope sensitivity, and (iii) the use of spin polarization technique. The examples provided deliver results that can hardly be obtained from e.g. X-ray diffraction.
\end{abstract}

PACS numbers: 61.41.+e, 61.43.-j, 61.05.fg

\section{Introduction}

Polymers are often referred to as model amorphous solids or glass-formers. Most often amorphous structures in polymers are thermodynamically stable. Indeed, due to morphologic complexity of their macromolecules, often branched or cross-linked, long-range crystal structures are a rarity. Much more likely, although still not frequent, are two-phase systems composed of small ordered regions embedded in an amorphous matrix. On a still smaller scale, a description of an amorphous polymer, relying solely on the principles of statistics, has long been refuted by both experimental evidence and theory [1]. Of interest here are therefore: (i) the arrangement of a single polymer chain, (ii) interchain interactions influencing the way polymer chains arrange. Both issues are difficult to study as: (i) the arrangement in question is highly influenced by the way particular sample was prepared, and (ii) they pose a major experimental challenge. Many still unresolved problems of local order in polymers focus researches' attention for reasons of fundamental interest in ground state of complex systems as well as due to practical tasks of e.g. designing polymeric materials to the needs of industry.

In what follows, we would refer to short range order if spatial correlations are limited to $c a .10 \mathrm{~nm}(100 \AA)$ in linear range. In general, short range order is not to be considered a piece of crystal structure confined to a small volume. On short distances the principles of crystal symmetry may easily be violated (cf. e.g. quasicrystals).

\section{Why neutrons?}

In any structure, be it a perfect crystal with long range translation symmetry, molecular objects such as micelles, clusters or polymer coils, the quantity being sought is the atomic spatial distribution, or the pair correlation function at $t \rightarrow \infty$. If such a structure is probed with a scattering technique of radiation whose wavelength is comparable to the distances of interest, then in the Fraunhofer (far-field) approximation, diffraction on a spatial distribution of matter is described by a Fourier transform of that distribution. For an ideal, infinite crystal lattice this yields a set of Dirac's delta functions multiplied by factors resulting from particular symmetry and physical characteristic of scattering objects (e.g. scattering efficiency or cross-section).

Here is a brief "wish list" to be satisfied before the best suited experimental technique is chosen. The probing radiation and the use thereof should be:

(i) Suitable for the investigation of an imperfectly ordered structure of organic material (dominating light atoms).

(ii) Capable of delivering information on correlation lengths, paracrystalinity, etc., e.g.

(iii) Capable of producing experimental results in absolute physical units rather than e.g. "scattering intensity". Such results can then be quantitatively compared with structure calculations from the first principles.

(iv) Of considerable penetration depth in order to probe the sample well beyond its surface layer.

(v) Non-destructive in order to enable the repeated measurements after long time or application of conplementary techniques.

(vi) Sensitive to isotopic substitution - a method helpful in hiding/enhancing signal from selected parts of complex systems. Very important for small angle scattering on complex or multiphase systems.

All the above postulates are met by neutron scattering techniques. In certain aspects neutron scattering proves superior to X-ray diffraction, especially regarding items (i), (iv), (vi) of the above list. 


\section{Diffuse scattering of polarized neutrons with polarization analysis}

However, neutron diffraction on polymers, much like on any organic matter, poses an experimental difficulty associated with exceptionally large incoherent scattering cross-section of hydrogen nuclei. Indeed, for hydrogen, the coherent cross-section amounts to 1.7567 barns, while the incoherent cross-section is as high as 80.26 barns. In case of a good crystalline order this would not be much of a problem. A highly imperfect and limited to a short distance structure of most polymers produces rather weak and broad peaks, which in case of hydrogen-rich polymer matter are accompanied by dominating incoherent scattering. The relative height of such peaks will usually be comparable to the effective statistical uncertainties. Fortunately, there exists a way to separate the two contributions to the scattering intensity, namely coherent and incoherent, at the level of the measuring instrument. It relies on the fact that the neutron spin may be either conserved or reversed in the act of scattering on a nucleus.

The interaction of the neutron spin with the nuclear spin $\hat{B}(\boldsymbol{I} \cdot \boldsymbol{\sigma})$ can be expressed in terms of the density matrix $\rho$, and the differential scattering cross-section $\frac{\mathrm{d} \sigma}{\mathrm{d} \Omega}$ which is ultimately linked to the measured intensity with and without spin flip. $\hat{B}$ represents the scattering matrix which describes the transformation of the neutron spin during the scattering process. Denoting the nuclear spin by $I$, the neutron spin by $\sigma$, and the scattering lengths by $b^{+}, b^{-}$the eigenvalues of the scattering length operator $\hat{B}$ are given by [2]:

$$
\begin{aligned}
& \hat{b}=\bar{b}+\frac{1}{2} b_{N} \boldsymbol{I} \cdot \boldsymbol{\sigma}, \quad b^{+}=\bar{b}+\frac{1}{2} b_{N} I, \\
& b^{-}=\bar{b}-\frac{1}{2} b_{N}(I+1),
\end{aligned}
$$

where

$$
\bar{b}=\frac{(I+1) b^{+}+I b^{-}}{2 I+1}, \quad b_{N}=\frac{2\left(b^{+}-b^{-}\right)}{2 I+1}=2 \hat{B} .
$$

A general expression for the differential cross-section in the density matrix formalism

$$
\frac{\mathrm{d} \sigma}{\mathrm{d} \Omega}=\operatorname{Tr}\left[\rho \hat{B}^{\dagger}\left(\boldsymbol{\sigma} \cdot \boldsymbol{I}^{\dagger}\right) \hat{B}(\boldsymbol{\sigma} \cdot \boldsymbol{I})\right]
$$

leads to a formulation of $\frac{\mathrm{d} \sigma}{\mathrm{d} \Omega}$ in terms of the polarization of the incoming beam $\boldsymbol{P}^{(\mathrm{in})}$ :

$$
\frac{d \sigma}{d \Omega}=\hat{B}^{\dagger} \hat{B}\left[I(I+1)+\mathrm{i} \boldsymbol{P}^{(\mathrm{in})}\left(\boldsymbol{I}^{\dagger} \times \boldsymbol{I}\right)\right] .
$$

Performing necessary averaging over spin and nuclear momentum orientations leads finally to the expression [2]:

and

$$
\frac{\mathrm{d} \sigma}{\mathrm{d} \Omega}=I^{\uparrow \uparrow}+I^{\uparrow \downarrow}=\hat{B}^{\dagger} \hat{B} I(I+1),
$$

$$
\begin{aligned}
& P_{\gamma}^{\text {(out) }} \frac{\mathrm{d} \sigma}{\mathrm{d} \Omega}=\frac{I_{\gamma}^{\uparrow \uparrow}-I_{\gamma}^{\uparrow \downarrow}}{I_{\gamma}^{\uparrow \uparrow}+I_{\gamma}^{\uparrow \downarrow}}\left(I_{\gamma}^{\uparrow \uparrow}+I_{\gamma}^{\uparrow \downarrow}\right)=I_{\gamma}^{\uparrow \uparrow}-I_{\gamma}^{\uparrow \downarrow} \\
& \quad=-\frac{1}{3} \hat{B}^{\dagger} \hat{B} I(I+1)
\end{aligned}
$$

where $\gamma$ denotes any defined direction in space, say $x, y$, or $z$. Solving the above equation for the intensity without spin flip $I^{\uparrow \uparrow}$ and for the intensity with spin flip $I^{\uparrow \downarrow}$, we obtain

$$
I^{\uparrow \uparrow}=\frac{1}{3}|B|^{2} I(I+1), \text { and } I^{\uparrow \downarrow}=\frac{2}{3}|B|^{2} I(I+1) .
$$

Hence, the outgoing scattering from unpolarized nuclear spins is always one third without, two thirds with flip of the neutron spin from the polarized beam.

Let us note that in this type of experiment the polarization $P_{\gamma}^{\text {(out) }} \frac{\mathrm{d} \sigma}{\mathrm{d} \Omega}=\ldots$ is not measured at all. Rather, we measure the cross-sections connecting the two neutron spin states $I^{\uparrow \uparrow}$ and $I^{\uparrow \downarrow}$. The experimental scattering intensities of $I^{\uparrow \uparrow}$ and $I^{\uparrow \downarrow}$ have to be corrected for a finite flipping ratio $R$ (measured separately using a quartz sample) and for multiple scattering

$$
\begin{aligned}
& I_{\text {corr }}^{\uparrow \uparrow}=I^{\uparrow \uparrow}-(R-1)^{-1}\left(I^{\uparrow \downarrow}-I^{\uparrow \uparrow}\right), \\
& I_{\text {corr }}^{\uparrow \downarrow}=I^{\uparrow \downarrow}+(R-1)^{-1}\left(I^{\uparrow \downarrow}-I^{\uparrow \uparrow}\right) .
\end{aligned}
$$

In scattering on an organic matter, the dominating incoherent process (with spin-flip) leads to the situation, where highly probable double scattering means double neutron spin-flip. When this happens, the given event becomes falsely classified as non-spin flip. The coherent scattering $I_{\mathrm{coh}}$, and incoherent scattering $I_{\text {inc }}$, are related to the measured spin-flip and non-spin-flip scattering via the relations

$$
I_{\mathrm{coh}}=I_{\mathrm{corr}}^{\uparrow \uparrow}-\frac{1}{2} I_{\mathrm{corr}}^{\uparrow \downarrow}, \quad \text { and } \quad I_{\mathrm{inc}}=\frac{3}{2} I_{\mathrm{corr}}^{\uparrow \downarrow} .
$$

Incoherent scattering may be made use of in order to normalize the coherent one. In this way the experiment produces coherent scattering cross-section expressed in e.g. barns per steradian per monomer or formula unit (this is often referred to as internal calibration):

$$
\frac{\partial \sigma_{\mathrm{coh}}}{\partial \Omega}=\frac{\sigma_{\mathrm{inc}}}{4 \pi} \frac{I_{\mathrm{coh}}}{I_{\mathrm{inc}}} .
$$

This technique has been used extensively to analyze the influence of various factors upon short range order in polymers. For example, poly (ethylene oxide) (PEO) complexed with lithium triflate $\left(\mathrm{LiSO}_{3} \mathrm{CF}_{3}\right)$ was studied at different concentrations, below and above $T_{\mathrm{g}}$ in order to determine the role lithium salt plays in changes of viscosity of the complexed polymer [3], Fig. 1.

Recently diffuse scattering of polarized neutrons with polarization analysis was applied to investigate residual strains in composites of porous $\mathrm{SiO}_{2}$ ceramic with poly(urea-urethane) elastomer [4]. The use of polarization analysis made it possible to detect and study very weak coherent scattering peaks from the elastomer synthesized inside the tiny pores of $\mathrm{SiO}_{2}$ ceramics. Residual strains were detected and measured based on inter-atomic distances in some but not all $\mathrm{SiO}_{2}+$ elastomer composites. Elastomer strains inside $\mathrm{SiO}_{2}$ pores were proved to develop in its soft segments, whose partly helical structure provided enough elasticity.

This method proved decisive in solving the puzzle of immiscibility/miscibility transition in lithium- 


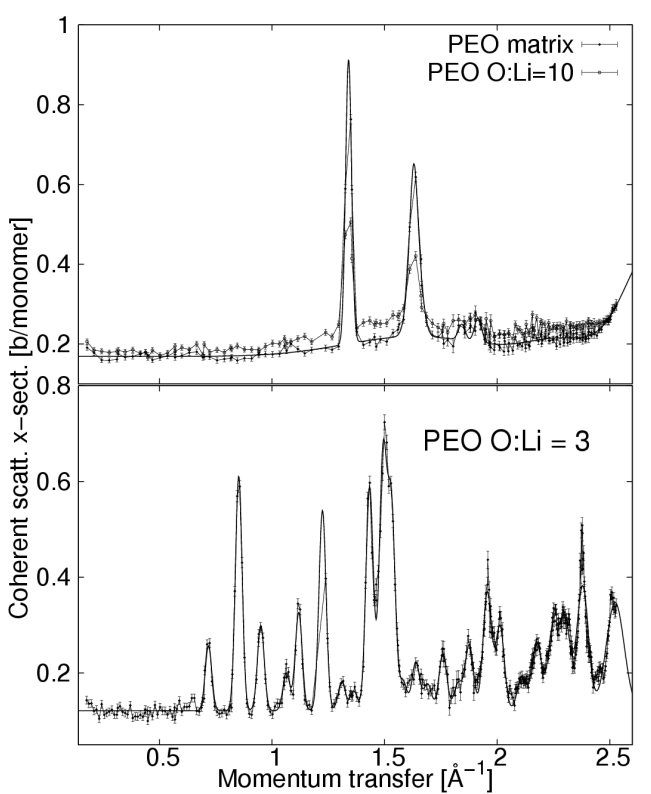

Fig. 1. The influence of lithium salt concentration upon short range order in $\mathrm{PEO} \cdot \mathrm{LiSO}_{3} \mathrm{CF}_{3}$ complexes. Spectra of diffuse scattering of polarized neutrons with polarization analysis. Absolute units through internal calibration. Measurements in ambient temperature.

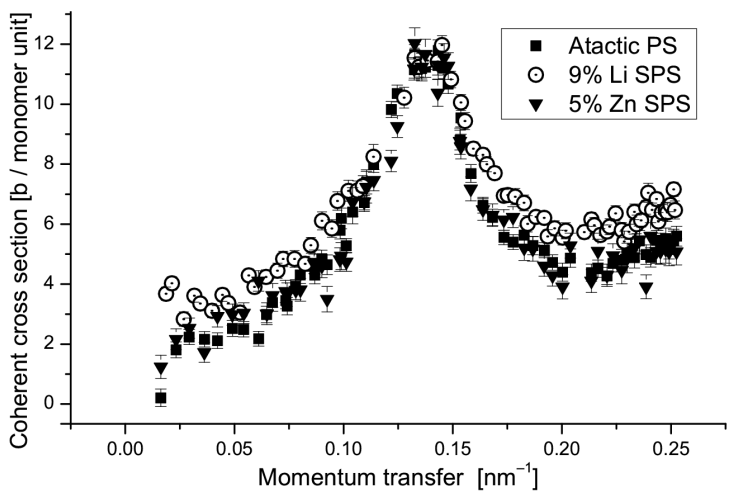

Fig. 2. The influence of counterion valency upon short range order in sulphonated polystyrene. Excess coherent scattering in lithium neutralized SPS most likely due to the process of $\pi$-type complexation.

-sulphonated polystyrene/polycarbonate blends, whose short range structure appears to depend upon the valency of counterion used to neutralize sulphonate groups [5], Fig. 2. Polymers usually do not mix well and various ways are sought to produce miscible blends of desired properties. Short range order is a known obstacle to the miscibility. On the other hand, ionic substitution occasionally facilitates miscibility due to repulsive electrostatic interactions [6].

Diffuse scattering of polarized neutrons with internal calibration provides results directly comparable with small- and intermediate angle neutron scattering. In

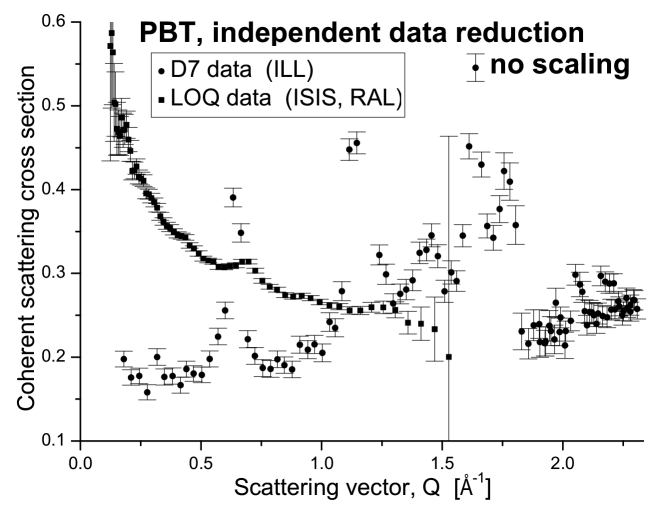

Fig. 3. Diffuse scattering of polarized neutrons with internal calibration produce data complementary to LOQ spectra taken with "high-Q" detector bank.

order to check this, an experiment was performed on poly(buthylene tereftalate) [7].

Partial overlap of scattering vector ranges was possible with the use of high-momentum-transfer detector bank available on small angle scattering instrument (LOQ) at the ISIS pulsed neutron source, RAL, Great Britain. The results of both D7 and LOQ experiments are presented in Fig. 3.

\section{Small angle neutron scattering}

The above example brings us to the aspects of short range order in polymers naturally accessible via the small angle scattering methods. Small angle neutron scattering (SANS) experiments routinely performed on dilute solutions provide information on size and shape of scattering objects such as coils or other aggregates. The idea of measurements on dilute solutions consists in effective eliminating interchain (or intercoil) interactions. SANS on bulk polymers deals with the other extreme: interchain interactions coexist with intrachain ones within the same space, and thus they contribute to the short range order. Rather complex picture of such interactions was revealed during investigation of the relationship between miscibility and short range order in blends of lithium-sulphonated polystyrene (Li-SPS) with polycarbonate $(\mathrm{PC})(\mathrm{Li}-\mathrm{SPS} / \mathrm{PC}=100 / 0 \%, 75 / 25 \%, 50 / 50 \%$ and $25 / 75 \%$ ) [5]. This blend is an upper critical solution temperature (UCST) system. It was studied by small angle neutron scattering both below and above critical mixing temperature with the aim to establish its phase behavior on microscopic scale. In the simplest case, a two-phase system (and thus microscopically immiscible) composed of regions of unidentified size and shape produces SANS spectrum which can be approximated by the so-called Debye-Bueche approximation

$$
I(Q)=\frac{A}{\left(1+a^{2} Q^{2}\right)^{2}} .
$$

Here, the parameter $a$ plays the role of a correlation length. In more complex cases of multiple-phase systems 
the formula (10) can be generalized to become e.g. [8, 9]:

$$
\begin{aligned}
& I(Q)=\frac{A_{1}}{\left(1+a_{1}^{2} Q^{2}\right)^{2}}+A_{2} \exp \left(-\frac{a_{2}^{2} Q^{2}}{4}\right), \\
& I(Q)=\frac{A_{1}}{\left(1+a_{1}^{2} Q^{2}\right)^{2}}+\frac{A_{2}}{\left(1+a_{2}^{2} Q^{2}\right)}
\end{aligned}
$$

or even

$$
I(Q) \sim \sum_{j} \frac{A_{j}}{\left(1+a_{j}^{2} Q^{2}\right)^{2}} .
$$

None of these models worked in the discussed case. Needless to say, formula (12), although theoretically acceptable, is useless in practical data analysis as it leads to an extremely ill-posed numerical problem [5]. Local density fluctuations in Li-SPS/PC blends most probably occur for two reasons: (i) a real many-phase system developing in the immiscible state, and (ii) ionic complexation effects, mediated via the metallic counterion. The nature of these fluctuations is complex enough to make the two-correlation-length Debye-Bueche approach hardly applicable. An almost perfect Debye-Bueche behavior was found, strangely enough, in pure sulphonated polystyrene, see Fig. 4. A two-phase behavior of this sample was attributed to ionic complexation ( $\pi$-type) phenomena.

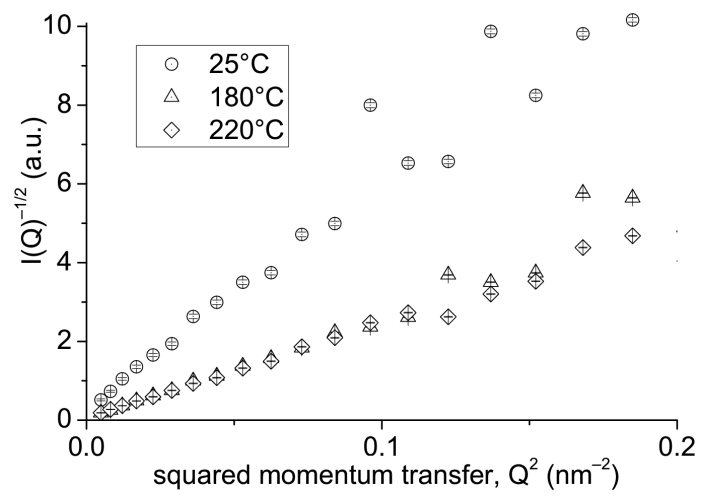

Fig. 4. Debye-Bueche plot of $9 \%$ Li-SPS revealing a two-phase system. Refer to Fig. 2 for explanation.

A good description of small angle scattering from $9 \% \mathrm{Li}-\mathrm{SPS} / \mathrm{PC}$ blends is achieved by assuming a microscopic mass-fractal structure of the material [10]:

$$
I(Q)=N V^{2}(\Delta \rho)^{2} P(Q, R) S(Q),
$$

where

$$
P(Q, R)=\left[\frac{3(\sin (Q R)-Q R \cos (Q R))}{(Q R)^{3}}\right]^{2},
$$

and

$$
\begin{aligned}
& S(Q)=\left\{\Gamma(D-1) \xi^{(D-1)}\left[1+\left(Q^{2} \xi^{2}\right)\right]^{\frac{1-D}{2}}\right. \\
& \quad \times \sin ((D-1) \arctan (Q \xi))\} / Q .
\end{aligned}
$$

Such an approach is not uncommon in polymers.
Branched or otherwise cross-linked structures often behave in a fractal-like way, showing limited scaling properties. However, in order to study such fractals one needs coherent scattering spectra collected over a very broad range of momentum transfer (scattering vector) values, typically stretching from conventional SANS through intermediate region up to wide angle neutron scattering (WANS) region (diffuse scattering) region [11]. Such studies were recently undertaken on pure atactic, isotactic and syndiotactic polystyrene, as well as on sulphonated atactic polystyrene with various sulphonation levels [12]. Preliminary calculations of fractal dimensionality revealed that these materials show surface rather than mass fractal behavior. Pure atactic polystyrene with its Porod exponent equal to $4.002( \pm 0.01)$ behaves as "smooth surface". On the other hand, sulphonation level of $8.4 \%$ yields a model rough surface image with the Porod exponent of 3.081 $( \pm 0.016)$.

Analysis of bulk polymer structures in terms of fractal models requires extreme care, since data collected over sufficiently broad range of scattering vector values require the use of a proper from of the cut-off function to account for the effects of limited scaling. The shape of this function has to be assumed and incorporated in data evaluation procedures.

\section{Conclusions}

The study of short range order in macroscopically amorphous or multi-phase polymers is not only interesting from the point of view of fundamental research (self-organization of complex matter). It is of utmost importance to the industry seeking materials of desired properties. Such are most polymer blends. Neutron scattering offers a wealth of experimental tools well suited to investigate these phenomena. Amongst them we count well-established and routinely used ones such as diffraction or SANS on solutions, as well as more sophisticated techniques (diffuse scattering of polarized neutrons with polarization analysis). The information they provide gradually improves the understanding of nanostructure of polymers [1].

\section{References}

[1] B. Gabryś, O. Schärpf, D.G. Peiffer, Ch. 4 in: Ionomers Characterization, Theory and Applications, Ed. S. Schlick, CRC Press, 1996, and references therein.

[2] G.L. Squires, Introduction to the Theory of Thermal Neutron Scattering, Dover Publ., Mineola 1997, Ch. 9.2.

[3] W. Zając, B.J. Gabryś, O. Schärpf, K.H. Andersend, E.E. Parsonage, Solid State Ionics 147, 213 (2002).

[4] W. Zajạc, A. Boczkowska, K. Babski, K. Kurzydłowski, P. Deen, Acta Materialia, DOI information: $10.1016 /$ j.actamat.2008.08.004.

[5] W. Zając, B.J. Gabryś, Phase Transit. 80, 501 (2007). 
[6] R. Tucker, B. Gabryś, W. Zając, K. Andersen, M.S. Kalhoro, R.A Weiss, Ch. 21 in: Scattering from Polymers: Characteriztion by X-rays, Neutrons and Light, Eds. P. Cebe, B.S. Hasiao, D.J. Lohse, ACS Series 739, Washington DC, 2000.

[7] S.M. King, unpublished data.

[8] Y. Wilson Cheung, R.S. Stein, G.D. Wignall, H.E. Yang, Macromolecules 26, 5365 (1993).
[9] D.W.M. Marr, Macromolecules 28, 8470 (1995).

[10] G. Beaucage, J. Appl. Crystallogr. 29, 134 (1996).

[11] R. Jullien, J. Phys. I (France) 2, 759 (1992).

[12] B.J. Gabryś, W. Zajạc, O. Schärpf, in preparation. 